\title{
Perfusion Deficits Detected by Arterial Spin-Labeling in Patients with TIA with Negative Diffusion and Vascular Imaging
}

\author{
X.J. Qiao, N. Salamon, D.J.J. Wang, R. He, M. Linetsky, B.M. Ellingson, and W.B. Pope
}

\begin{abstract}
BACKGROUND AND PURPOSE: A substantial portion of clinically diagnosed TIA cases is imaging-negative. The purpose of the current study is to determine if arterial spin-labeling is helpful in detecting perfusion abnormalities in patients presenting clinically with TIA.
\end{abstract}

MATERIALS AND METHODS: Pseudocontinuous arterial spin-labeling with 3D background-suppressed gradient and spin-echo was acquired on 49 patients suspected of TIA within 24 hours of symptom onset. All patients were free of stroke history and had no lesion-specific findings on general MR, DWI, and MRA sequences. The calculated arterial spin-labeling CBF maps were scored from 1-3 on the basis of presence and severity of perfusion disturbance by 3 independent observers blinded to patient history. An age-matched cohort of 36 patients diagnosed with no cerebrovascular events was evaluated as a control. Interobserver agreement was assessed by use of the Kendall concordance test.

RESULTS: Scoring of perfusion abnormalities on arterial spin-labeling scans of the TIA cohort was highly concordant among the 3 observers $(W=0.812)$. The sensitivity and specificity of arterial spin-labeling in the diagnosis of perfusion abnormalities in TIA was $55.8 \%$ and $90.7 \%$, respectively. In $93.3 \%$ (70/75) of the arterial spin-labeling CBF map readings with positive scores ( $\geq 2)$, the brain regions where perfusion abnormalities were identified by 3 observers matched with the neurologic deficits at TIA onset.

CONCLUSIONS: In this preliminary study, arterial spin-labeling showed promise in the detection of perfusion abnormalities that correlated with clinically diagnosed TIA in patients with otherwise normal neuroimaging results.

ABBREVIATIONS: ASL = arterial spin-labeling; ATT = arterial transit time; $\mathrm{PCT}=$ contrast-enhanced perfusion CT; PLD = postlabel delay

T IA is traditionally defined as the sudden onset of a neurologic deficit(s) as a result of transient ischemia of the eloquent brain, which resolves completely within 24 hours. TIA is presumed to have a vascular cause and is considered to be an important risk factor for stroke. ${ }^{1,2}$ In practice, the unequivocal diagnosis of TIA has been limited by the high percentage of patients who had suspected neurologic deficits, but lacked confirmatory imaging. This contributes to lack of agreement in the diagnosis of TIA among practicing physicians ${ }^{3}$ and has led to proposals advocating the incorporation of imaging data, in addition to clinical findings, in the diagnosis of TIA. For instance, this new paradigm has been adopted by American Stroke Association. ${ }^{4}$ In addition to diagno-

Received September 7, 2012; accepted after revision January 31, 2013.

From the Departments of Radiological Sciences (X.J.Q., N.S, M.L., B.M.E., W.B.P.), and Neurology (D.J.J.W.), David Geffen School of Medicine; and Department of Biostatistics (R.H.), School of Public Health, UCLA, Los Angeles, California.

Please address correspondence to Whitney B. Pope, MD, PhD, Department of Radiological Sciences, David Geffen School of Medicine, UCLA Medical Center, 10833 Le Conte Ave, BL-428/CHS Los Angeles, CA 90095-1721; e-mail: wpope@mednet.ucla.edu - Indicates open access to non-subscribers at www.ajnr.org

三 indicates article with supplemental on-line table

http://dx.doi.org/10.3174/ajnr.A3551 sis, detection of malperfused tissue is considered to be important in understanding the extent of the initial perfusion deficit and may be helpful in guiding therapeutic decisions.

SPECT and contrast-enhanced perfusion CT (PCT) have been previously used to investigate perfusion disturbances in clinical TIA cases. ${ }^{5-7}$ However, these CT-based imaging techniques have several disadvantages including invasiveness, reliance on radioactive materials, adverse reactions to contrast material, as well as technical difficulties in covering large brain volumes. ${ }^{5,8,9}$ More recently, the introduction of advanced MR imaging technology has enabled better visualization of ischemic cerebral regions corresponding both anatomically and temporally to symptoms of TIA. For example, DWI results were positive for acute ischemic lesions in $24 \%-40 \%$ of patients referred for evaluation of TIA. ${ }^{10-14}$ In addition, contrast-based PWI has shown promise in the identification of abnormalities in approximately one-third of patients with TIA, some of whom had negative DWI results. ${ }^{15,16}$ Nonetheless, a substantial portion of patients diagnosed with TIA clinically lack confirmatory imaging findings.

The arterial spin-labeling (ASL) technique provides CBF measurements without the use of a contrast agent. ASL has shown 

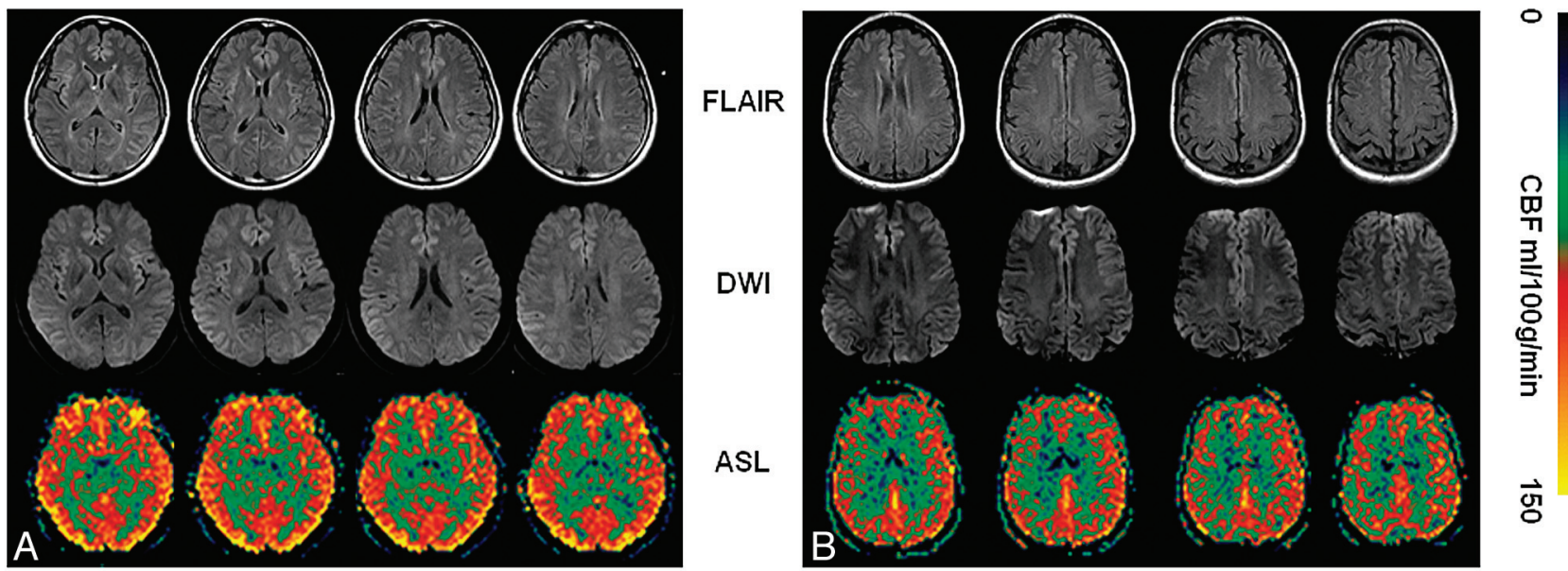

FIG 1. Representative cases from the control cohort. A, A 50-year-old woman was evaluated for dizziness after an automobile crash. No specific findings are reported on the standard MR, DWI, and MRA tests. ASL reading scores are rated 1, 1, 1 by 3 raters. B, A 49-year old woman was evaluated for a chronic headache of unknown cause. No specific findings are reported on the standard MR, DWI, and MRA tests. ASL reading scores are rated 2, 2, 2 by 3 raters.

promise in clinical studies of ischemic pathologic conditions, such as acute stroke ${ }^{17-19}$ and large-artery occlusion. ${ }^{20,21}$ In a limited number of published studies, ASL has demonstrated a high sensitivity for detection of minor perfusion alterations in patients with TIA. ${ }^{12,22}$ With the goal of establishing a vascular cause of TIA symptoms, the current study investigated the value of ASL in the detection of hypoperfusion abnormalities in a TIA cohort free of stroke history and without confirmatory findings on standard MR, DWI, and MRA examinations.

\section{MATERIALS AND METHODS \\ Patients}

From an ongoing prospective registry of consecutive patients evaluated for suspected TIA from July 2010 to July 2011 at our medical center, imaging data were selected from all patients who had 1) transient neurologic symptoms judged by clinical neurologists at the end of the evaluation to have a possible vascular cause; 2) no stroke history; 3) MR images performed within 24 hours of symptom onset; and 4) nonspecific findings on general MR, DWI, and MRA examinations. ASL imaging was used only in this retrospective analysis but not in the clinical evaluation of the patients. The selected patients were then followed up in clinic for complaints of any new neurologic symptoms until December 31, 2011, when the study was terminated. A control cohort of agematched patients ( $>40$ years old), who had received a brain MR imaging scan because of neurologic symptoms thought to be irrelevant to vascular events, was selected from another ongoing prospective registry of consecutive patients during the same period (Fig 1). For all selected patients in the TIA cohort (Fig 2), $\mathrm{ABCD}^{2}$ scores were generated based on the presence of pre-existing conditions. ${ }^{10,11}$ This study was approved by our institutional review board and was Health Insurance Portability and Accountability Act compliant.

\section{MR Imaging Protocols}

MR imaging scans were performed on a 1.5T Avanto or 3T Tim Trio system (Siemens, Erlangen, Germany) by use of a 12-channel head coil. The stroke imaging protocol included DWI, FLAIR, and gradient recalled-echo. ASL scans were performed by use of pseudocontinuous pulse sequence with background-suppressed $3 \mathrm{D}$ gradient and spin-echo readout (labeling pulse duration, 1.5 seconds; postlabel delay [PLD], 2 seconds; no flow-crushing gradient; FOV, $22 \mathrm{~cm}$; matrix size, $64 \times 64$; number of 5 -mm sections, 26; generalized autocalibrating partially parallel acquisition, 2; TE, $22 \mathrm{~ms}$; TR, $4000 \mathrm{~ms}$; with 30 pairs of tag and control volumes acquired within a total of 4 minutes). ${ }^{23,24}$

\section{ASL Postprocessing and Evaluation}

ASL images were corrected for motion; pair-wise subtracted between labeled and unlabeled images; and averaged to generate mean difference images, or ASL CBF maps. ASL CBF maps of patients with TIA $(n=49)$ and control patients $(n=36)$ were scored by 3 independent observers ( 2 board-certified neuroradiologists and 1 nonradiologist physician experienced in MR imaging processing) blinded to patient history. The reading scores, ranging from a scale of $1-3$, reflected the presence and severity of the perfusion disturbance (usually hypoperfusion in this study): "1" for normal (no readable altered perfusion), " $\geq 2$ " being defined as "positive" readings indicating recognizable ischemic lesions (" 2 " for subtle abnormalities only identified by careful study of ASL CBF maps, and “ 3 ” for prominent perfusion deficits easily identified on ASL CBF maps). The laterality of the perfusion disturbance was recorded in patients with hemispheric TIA for comparison with neurologic symptoms at onset. ASL reading scores were compared between patients grouped by field strength (1.5T vs $3 \mathrm{~T}$ ) of the scanner on which ASL images were obtained.

\section{Statistical Analysis}

The agreement in reading scores from 3 observers $(n=49 \times 3$ for the TIA cohort; $n=36 \times 3$ for the control cohort) was assessed by use of a Kendall concordance test. Kendall $W$, also known as the Kendall coefficient of concordance, was calculated to evaluate the degree of consensus. Pooled observations from all 3 observers ( $n=49 \times 3$ for the TIA cohort; $n=36 \times 3$ for the control cohort) were recorded in a $2 \times 2$ contingency table reflecting the frequency of true-positive, true-negative, false-positive, and false- 

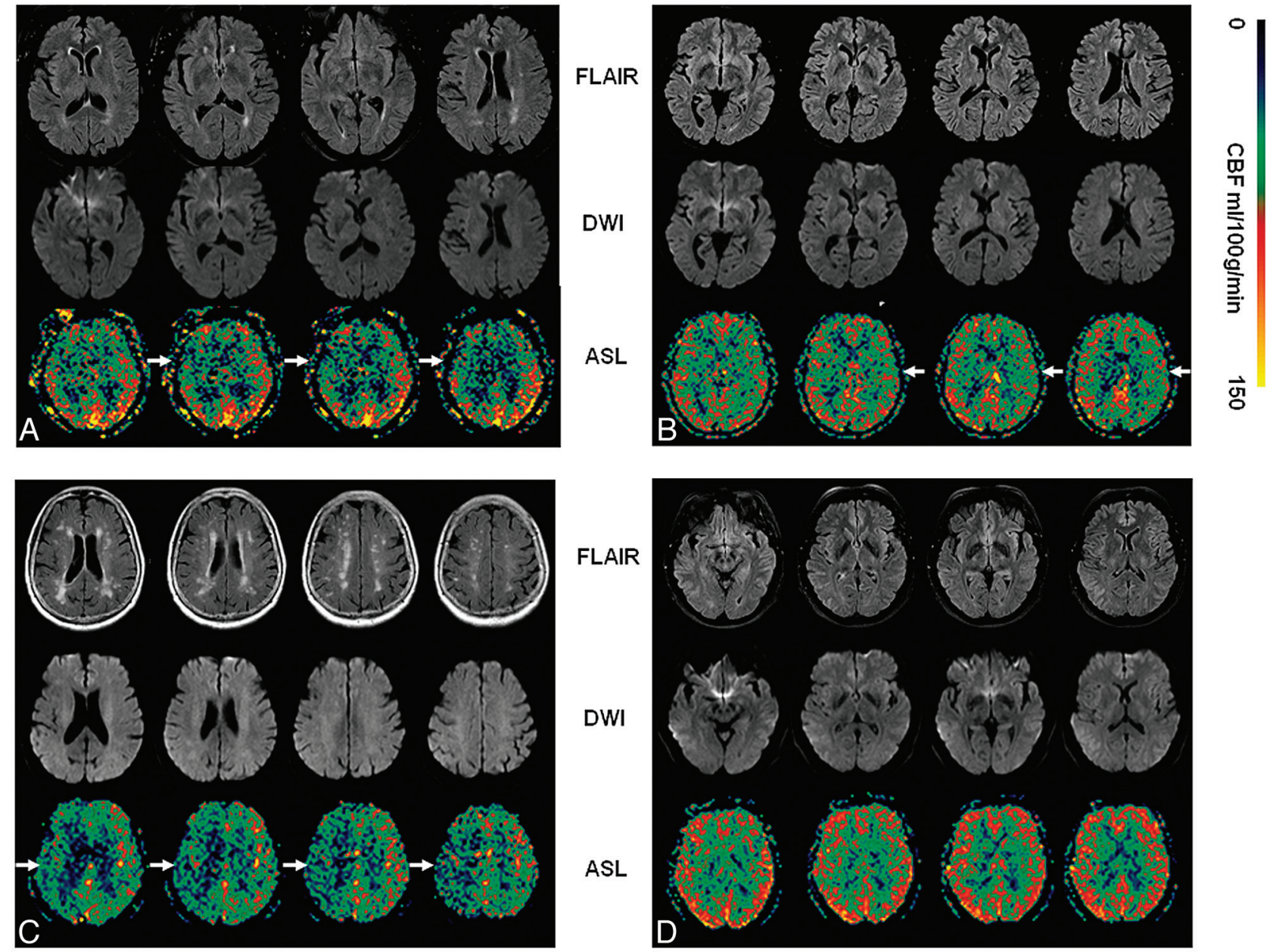

FIG 2. Representative cases from the TIA cohort. A, A 75-year-old woman with a history of hypertension and diabetes had an acute onset of transient blurry vision, slurred speech, dysarthria, and word-finding difficulties that lasted for $20-30$ minutes. $A B C D^{2}$ score is 5 . The standard MR imaging results demonstrate a few nonspecific T2/FLAIR hyperintensities in the cerebral white matter bilaterally. DWI and MRA study results are normal. ASL CBF map shows perfusion deficits in the right MCA region (arrow). ASL reading scores are rated 2, 2, 2 by 3 raters. B, A 57-year old woman with a history of hypertension had right facial droop and right arm numbness for 6 hours. ABCD ${ }^{2}$ score is 3. Nonspecific T2/FLAIR hyperintensities are seen in the cerebral white matter. DWI and MRA study results are normal. ASL CBF map shows perfusion deficits in the regions of the left MCA and left posterior cerebral artery (arrow). ASL scores are rated 2, 2, 2 by 3 raters. C, A 80-year old woman with a history of hyperlipidemia had transient global amnesia, left-sided sensory deficit, and a positive Babinski sign on the left side for 6 hours. ABCD score $^{2}$ is 3. Scattered T2/FLAIR hyperintensities are seen in the periventricular and subcortical white matter on standard MR imaging. DWI and MRA study results are normal. ASL CBF map shows perfusion deficits in the right MCA region (arrow). ASL scores are rated 3, 3, 3 by 3 raters. D, A 57-year-old woman with a history of hypertension had right facial droop and slurred speech for more than 1 hour. $A B C D^{2}$ score was 4 . The standard MR, DWI, and MRA study results are normal. ASL reading scores are rated 1, 1, 1 by 3 raters.

negative observations as rated by ASL CBF maps compared with the clinical diagnosis of TIA (reference standard). A true-positive result was obtained from a patient clinically diagnosed with TIA with an ASL CBF map reading score $\geq 2$. Conversely, a true-negative result was obtained from a control patient with an ASL CBF map reading score of 1 . Sensitivity and specificity were calculated. A $t$ test was used to compare ASL reading scores between imaging performed on scanners with different magnetic field strengths (1.5T vs $3 \mathrm{~T})$. The significance level was defined as $P<.05$ (2-sided).

\section{RESULTS}

\section{Patient Characteristics}

From July 2010 to July 2011 at our medical center, we recorded 165 patients who were suspected of having a TIA, among whom $67(40.6 \%)$ had a history of ischemic stroke. In patients who tested negative for a stroke history (98/165), 23 patients $(23.4 \%)$ had positive DWI findings, and 30 patients (30.6\%) had vascular lesions identified by MRA. In this particular subgroup, 4 patients (4.1\%) showed positive findings on both DWI and MRA. Finally, 49 patients (50.0\%) were "imaging negative" and were selected for our current analysis. Until December 31, 2011, the selected imaging-negative patients with TIA were followed up in clinic for 164538 days (median, 370 days) for complaints of new neurologic symptoms. Patients did not receive follow-up MR imaging.

Another cohort of 36 age-matched patients ( $>40$ years old), who had received a brain MR imaging scan because of neurologic symptoms thought to be irrelevant to vascular events, was selected from another ongoing prospective registry of consecutive patients during the same period as a control (On-Line Table).

Table 1 shows baseline characteristics of "imaging-negative" patients with TIA $(n=49)$ at symptom onset and control patients 
Table 1. Basic characteristics of patient cohorts

\begin{tabular}{|c|c|c|}
\hline \multirow[b]{2}{*}{ Characteristics } & \multicolumn{2}{|c|}{ Cohort } \\
\hline & $\operatorname{TIA}^{\mathrm{a}}(n=49)$ & Control $(n=36)$ \\
\hline Age $(\text { mean } \pm S D y)^{b}$ & $65.6 \pm 14.6$ & $60.2 \pm 12.2$ \\
\hline Sex $(F / M)$ & $27 / 22$ & $24 / 12$ \\
\hline MR imaging delay median $(h)^{c}$ & 6.5 & $\mathrm{~N} / \mathrm{A}$ \\
\hline$A B C D^{2}$ score median & 4 & N/A \\
\hline Prior TIA, $n(\%)^{d}$ & $9(18.4)$ & $0(0)$ \\
\hline Coronary artery disease, $n(\%)^{\mathrm{d}}$ & $10(20.4)$ & $3(8.3)$ \\
\hline Atrial fibrillation, $n(\%)^{\mathrm{b}}$ & $1(2.0)$ & $1(2.8)$ \\
\hline Hyperlipidemia, $n(\%)^{\mathrm{d}}$ & $24(49.0)$ & $5(13.9)$ \\
\hline Hypertension, $n(\%)^{d^{d}}$ & $31(63.3)$ & $7(19.4)$ \\
\hline Diabetes, $n(\%)^{\mathrm{d}}$ & $9(18.4)$ & $2(5.6)$ \\
\hline
\end{tabular}

${ }^{a}$ Diagnosis of TIA was made clinically without ASL imaging.

${ }^{\mathrm{b}}$ No significant difference exists between the groups.

c Based on 40 patients, data were not available for 9 patients.

${ }^{\mathrm{d}}$ Significant differences exist between the groups, $P<.001$.

Table 2. Sensitivity and specificity of ASL CBF map reading ${ }^{a}$

\begin{tabular}{lccr}
\hline & \multicolumn{3}{c}{ Cohort } \\
\cline { 2 - 3 } & TIA $^{\mathrm{b}}$ & Control & \\
\hline ASL positive $^{\mathrm{c}}$ & 82 & 10 & 92 \\
ASL negative $^{\mathrm{d}}$ & 65 & 98 & 163 \\
& 147 & 108 & \\
& Sensitivity, 55.78\% & Specificity, 90.73\% \\
\hline
\end{tabular}

${ }^{a}$ Based on pooled data from 3 independent observers, $n=49$ for TIA cohort and $n=$ 36 for control cohort, with scores of $\geq 2$ being defined as "positive" readings indicating recognizable ischemic lesions $(82 / 147=49 \times 3$ for the TIA cohort, and 10/108 $=$ $36 \times 3$ for the control cohort).

${ }^{\mathrm{b}}$ Diagnosis of TIA was made clinically without ASL imaging.

'Defined as a reading score of 2 or 3.

${ }^{\mathrm{d}}$ Defined as a reading score of 1 .

( $n=36$ ) on MR imaging dates. Patients with a clinical diagnosis of TIA $(n=49)$ but no prior stroke and positive MR imaging findings, had a sudden onset of neurologic deficits that lasted anywhere from seconds to hours. In all cases, symptoms resolved by the time of MR scanning, which was performed within 1-11 hours after the initial onset of symptoms. The TIA and control cohorts showed no significant difference in mean age but were significantly different for pre-existing conditions associated with stroke risk, including the incidence of TIA, coronary heart disease, hyperlipidemia, hypertension, and diabetes mellitus. The TIA cohort has a median $\mathrm{ABCD}^{2}$ score of 4 . All patients in the TIA cohort were followed up for an average period of 1 year (range, 164-538 days), and none of the patients was found to have a post-TIA stroke on the basis of absence of complaints of any new neurologic symptoms.

\section{Evaluation of ASL CBF Maps}

Results suggested relatively high concordance scores (Kendall concordance test), indicating good agreement in evaluating ASL CBF maps of both TIA ( $W=0.812)$ and control cohorts $(W=$ 0.642 ) among 3 observers. With scores of $\geq 2$ being defined as "positive" readings indicating recognizable ischemic lesions (82/ $147=49 \times 3$ for the TIA cohort, and $10 / 108=36 \times 3$ for the control cohort), the sensitivity and specificity of the ASL CBF map in the diagnosis of TIA were $55.8 \%$ (95\% CI, 0.49-0.63) and 90.7\% (95\% CI, 0.87-0.95), respectively (Table 2). The magnetic field strength (23 patients on 1.5T, 26 patients on $3 \mathrm{~T}$ in the TIA cohort; 17 patients on $1.5 \mathrm{~T}, 19$ patients on $3 \mathrm{~T}$ in the control cohort) was not associated with a significant difference in mean reading scores of ASL CBF maps ( $t$ test). In the TIA cohort, 44 $(89.8 \%)$ of 49 patients presented with hemispheric symptoms, such as focal weakness and aphasia. In this subgroup of patients with hemispheric TIA and positive ASL CBF map readings (score $\geq 2$ ), $93.3 \%$ (70/75 positive readings) of the cases showed brain regions where the hypoperfusion identified matched the laterality of the neurologic symptoms at TIA onset. In the rest of the observations (5/75), hyperperfusion was characterized in brain regions corresponding to transient neural deficits during the TIA attacks.

\section{DISCUSSION}

The widespread adoption of newer advanced MR imaging, such as DWI, has significantly improved the characterization of ischemic lesions in patients with TIA. However, the diagnosis of TIA in a substantial proportion of cases remains purely clinically based, as no corroborating imaging findings exist by standard MR imaging. Therefore, it would be of potential value to identify imaging technologies with a high sensitivity for the detection of minor cerebral perfusion abnormalities, to more confidently establish the diagnosis of TIA in clinical practice. ASL has emerged as such an imaging technique with a purported high sensitivity for the detection of perfusion abnormalities in both TIA and acute stroke. ${ }^{17-19,22}$ In our current study, we explored the value of ASL in the detection of perfusion deficits in a cohort of clinically diagnosed patients with TIA with unremarkable standard MR imaging results. To avoid confounding factors that are known to produce positive findings on ASL images, we excluded patients with a history of stroke and those with any brain lesions that can be identified on DWI and MRA sequences. As a preliminary investigation of the value of ASL in TIA, our current analysis was limited by small study size and discrepancy in the sex ratio between TIA and control cohorts (Table 1). The power of the study was further affected by the gap in the median age of the TIA and control cohorts (70.7 years and 61.4 years, respectively), though there was no significant difference in the mean age of the patient cohorts ( 65.6 years and 60.2 years, respectively; $P>.05, t$ test).

We found that ASL provided imaging evidence of disturbed perfusion (this study focused on hypoperfusion) in 55.8\% of imaging-negative TIA cases, as judged by the standard imaging protocols including DWI and MRA. ASL CBF map reading also showed a high specificity (90.7\%) for abnormal perfusion in our patient cohort. Positive ASL CBF map readings showed that the location of the hypoperfusion was consistent with the neurologic symptoms at TIA onset in $93.3 \%$ (70/75) of the total observations. These findings support the hypothesis that ASL is a feasible and practical method for detection of perfusion abnormalities, with good sensitivity in patients with a clinical diagnosis of TIA. The evaluation of ASL CBF maps demonstrated good agreement in reading scores from 3 observers ( $W=0.812$ for TIA and $W=0.642$ for control), suggesting that ischemic lesions in ASL CBF maps could be consistently recognized by different readers, and thus supporting the conclusion that ASL has good reliability as well. In practice, ASL has the advantages of being contrast-free and requiring a relatively short scan time $(<4$ minutes in our protocol), which may allow this technique to be widely used in the clinical management of TIA and other clinical emergencies that mimic it. 
Prospective studies have shown a high risk for stroke within the immediate hours and days after TIA. ${ }^{2}$ In our current study, we followed up on the TIA cohort for an average period of 1 year (range, 164-538 days), and none of the patients experienced a post-TIA stroke. The reported factors correlating with the risk for post-TIA stroke include $\mathrm{ABCD} / \mathrm{ABCD}^{2}$ scores $^{10,11,25}$ and positive MR imaging findings on $\mathrm{DWI}^{25-29}$ and MRA. ${ }^{28-30}$ As a clinical score based on the presence of pre-existing conditions to determine the risk for stroke after a TIA, the value of $\mathrm{ABCD} / \mathrm{ABCD}^{2}$ scores in the prediction of post-TIA stroke risk have been controversial and vary greatly by patient cohort and clinical protocol. ${ }^{10,11,25,30}$ Our TIA cohort had a high median $\mathrm{ABCD}^{2}$ score of 4 ; however, none of the patients appeared to have a stroke during the average 1-year follow-up period, though silent infarcts could have gone undetected (as patients had routine clinical follow-up but no additional MR imaging).

The lack of clinically evident strokes in our TIA cohort may be surprising, but it may suggest that the negative standard imaging being used as a criterion for study inclusion could be associated with reduced stroke risk. Although the power of the current analysis was limited by a relatively small cohort size, we found that patients with TIA with positive findings on DWI and MRA are more likely to display readable abnormalities on ASL than imaging-negative cases used in the current study (data not shown), raising the question of whether ASL data should be included in criteria for the evaluation of stroke risk after TIA onset. Better characterization of such a patient cohort may help to clarify the value of $\mathrm{ABCD} / \mathrm{ABCD}^{2}$ scores in the prediction of post-TIA stroke. Specifically, future studies of much larger sizes will be necessary to determine stroke risks in these patient groups: those with TIA with MR imaging abnormalities (including DWI and MRA), those with TIA with ASL abnormalities only, and those with TIA with no MR imaging or ASL abnormalities.

Low SNR and low spatial resolution are major limitations of ASL. The SNR is directly proportional to voxel size and the PLD time, which is defined as the duration between labeling of the spins and acquisition of the images, and is typically between 1.5 and 2.0 seconds. Longer PLDs ( $>2$ seconds) could help to improve $\mathrm{CBF}$ quantification, however, at the cost of a further decrease in SNR. ${ }^{31}$ In the current analysis, a single PLD of 2.0 seconds was used, resulting in the detection of focal hypoperfusion in $55.8 \%$ of imaging-negative patients with TIA. Considering the universal existence of multiple conditions in patients with TIA that related to vascular pathogenesis, arterial transit time (ATT) could vary greatly among individual patients. Therefore, $1 \mathrm{calcu}-$ lated CBF map on the basis of a single PLD might not be the best approach to accurately reflect the perfusion status in these patients. In this case, an option would be to obtain images at multiple PLDs, which offers the potential to quantify $\mathrm{ATT}^{32}$ and effectively map the inflow of label into the tissue. ${ }^{20,33}$ In future studies, we plan to perform ASL scans by using multiple PLDs and calculate ATT-adjusted CBF maps, aiming to improve the sensitivity in the detection of minor perfusion changes in patients with TIA.

\section{CONCLUSIONS}

Our results suggest the potential value of ASL perfusion data in the detection of abnormalities in patients with TIA, which may help improve the diagnostic certainty in otherwise imaging-negative patients with TIA.

Disclosures: Danny JJ Wang—RELATED: Grant: NIMH.* Whitney Pope—UNRELATED: Consultancy: Genentech. *Money paid to institution.

\section{REFERENCES}

1. Rothwell PM, Giles MF, Flossmann E, et al. A simple score (ABCD) to identify individuals at high early risk of stroke after transient ischaemic attack. Lancet 2005;366:29-36

2. Giles M, Rothwell P. Risk of stroke early after transient ischaemic attack: A systematic review and meta-analysis. Lancet Neurol 2007;6:1063-72

3. Castle J, Mlynash M, Lee K, et al. Agreement regarding diagnosis of transient ischemic attack fairly low among stroke-trained neurologists. Stroke 2010;41:1367-70

4. Easton JD, Saver JL, Albers GW, et al. Definition and evaluation of transient ischemic attack: a scientific statement for healthcare professionals from the American Heart Association/American Stroke Association Stroke Council; Council on Cardiovascular Surgery and Anesthesia; Council on Cardiovascular Radiology and Intervention; Council on Cardiovascular Nursing; and the Interdisciplinary Council on Peripheral Vascular Disease. Stroke 2009;40:2276-93

5. Laloux P, Jamart J, Meurisse H, et al. Persisting perfusion defect in transient ischemic attacks: a new clinically useful subgroup? Stroke 1996;27:425-30

6. Lu J, Li KC, Hua Y. Primary study on imaging in transient ischemic attack. Chin Med J 2005;118:1812-16

7. Prabhakaran S, Patel SK, Samuels J, et al. Perfusion computed tomography in transient ischemic attack. Arch Neurol 2011;68:85-89

8. Eastwood JD, Lev MH, Wintermark M, et al. Correlation of early dynamic CT perfusion imaging with whole-brain MR diffusion and perfusion imaging in acute hemispheric stroke. AJNR Am J Neuroradiol 2003;24:1869-75

9. Koenig M, Klotz E, Luka B, et al. Perfusion CT of the brain: diagnostic approach for early detection of ischemic stroke. Radiology 1998;209:85-93

10. Merwick A, Albers GW, Amarenco P, et al. Addition of brain and carotid imaging to the $\mathrm{ABCD}^{2}$ score to identify patients at early risk of stroke after transient ischaemic attack: a multicentre observational study. Lancet Neurol 2010;9:1060-69

11. Giles MF, Albers GW, Amarenco P, et al. Early stroke risk and ABCD2 score performance in tissue- vs time-defined TIA: a multicenter study. Neurology 2011;77:1222-28

12. Zaharchuk G, Olivot JM, Fischbein NJ, et al. Arterial spin labeling imaging findings in transient ischemic attack patients: comparison with diffusion- and bolus perfusion-weighted imaging. Cerebrovasc Dis 2012;34:221-28

13. Ay H, Arsava EM, Johnston SC, et al. Clinical- and imaging-based prediction of stroke risk after transient ischemic attack: the CIP model. Stroke 2009;40:181-86

14. Sylaja PN, Coutts SB, Krol A, et al. When to expect negative diffusion-weighted images in stroke and transient ischemic attack. Stroke 2008;39:1898-900

15. Krol AL, Coutts SB, Simon JE, et al. Perfusion MRI abnormalities in speech or motor transient ischemic attack patients. Stroke 2005;36:2487-89

16. Mlynash M, Olivot JM, Tong DC, et al. Yield of combined perfusion and diffusion MR imaging in hemispheric TIA. Neurology 2009;72:1127-33

17. Chalela JA, Alsop DC, Gonzalez-Atavales JB, et al. Magnetic resonance perfusion imaging in acute ischemic stroke using continuous arterial spin labeling. Stroke 2000;31:680-87

18. Wang DJ, Alger JR, Qiao JX, et al. The value of arterial spinlabeled perfusion imaging in acute ischemic stroke: comparison

AJNR Am J Neuroradiol 34:2125-30 Nov 2013 www.ajnr.org 2129 
with dynamic susceptibility contrast-enhanced MRI. Stroke 2012;43:1018-24

19. Zaharchuk G. Arterial spin label imaging in acute ischemic stroke and transient ischemic attack. Neuroimaging Clin N Am 2011;21: 285-301, $x$

20. Hendrikse J, van Osch MJ, Rutgers DR, et al. Internal carotid artery occlusion assessed at pulsed arterial spin-labeling perfusion MR imaging at multiple delay times. Radiology 2004;233:899-904

21. van Laar PJ, Hendrikse J, Klijn CJ, et al. Symptomatic carotid artery occlusion: flow territories of major brain-feeding arteries. Radiology 2007;242:526-34

22. MacIntosh BJ, Lindsay AC, Kylintireas I, et al. Multiple inflow pulsed arterial spin-labeling reveals delays in the arterial arrival time in minor stroke and transient ischemic attack. AJNR Am J Neuroradiol 2010;31:1892-94

23. Fernández-Seara MA, Edlow BL, Hoang A, et al. Minimizing acquisition time of arterial spin labeling at 3T. Magn Reson Med 2008;59:1467-71

24. Wu WC, Fernandez-Seara M, Detre JA, et al. A theoretical and experimental investigation of the tagging efficiency of pseudo-continuous arterial spin labeling. Magn Reson Med 2007;58:1020-27

25. Ghia $\mathrm{D}$, Thomas $\mathrm{P}$, Cordato $\mathrm{D}$, et al. Low positive predictive value of the $\mathrm{ABCD} 2$ score in emergency department transient ischaemic attack diagnoses: the South Western Sydney tRansient Ischaemic Attack study. Intern Med J 2012;42:913-18
26. Crisostomo RA, Garcia MM, Tong DC. Detection of diffusionweighted MRI abnormalities in patients with transient ischemic attack: correlation with clinical characteristics. Stroke 2003; 34:932-37

27. Ay H, Oliveira-Filho J, Buonanno FS, et al. 'Footprints' of transient ischemic attacks: a diffusion-weighted MRI study. Cerebrovasu Dis 2002;14:177-86

28. Calvet D, Touzé E, Oppenheim C, et al. DWI lesions and TIA etiology improve the prediction of stroke after TIA. Stroke 2009; 40:187-92

29. Ssi-Yan-Kai G, Nasr N, Faury A, et al. Intracranial artery stenosis or occlusion predicts ischemic recurrence after transient ischemic attack. AJNR Am J Neuroradiol 2013;34:185-90

30. Hotter BA, Lechner JM, Nolte $\mathrm{CH}$, et al. $\mathrm{ABCD}(2)$ as a screening tool for cerebral infarction on stroke MRI? Eur Neurol 2012;67:315-20

31. Alsop DC, Detre JA. Reduced transit time sensitivity in noninvasive magnetic resonance imaging of human cerebral blood flow. J Cereb Blood Flow Metab 1996;16:1236-49

32. Buxton RB, Frank LR, Wong EC, et al. A general kinetic model for quantitative perfusion imaging with arterial spin labeling. Magn Reson Med 1998;40:383-96

33. Petersen ET, Lim T, Golay X. Model-free arterial spin labeling quantification approach for perfusion MRI. Magn Reson Med 2006;55: $219-32$ 